\title{
CaNOP 3U CubeSat Electrical Power System: Power Tests
}

\author{
Austin Weber \\ Carthage College, Kenosha WI
}

\begin{abstract}
:
The Canopy Near Infrared Observing Project (CaNOP) consists of a 3U CubeSat nanosatellite funded through WSGC. Its mission objective is to capture multispectral images across global forests, similar to that of the Landsat missions. In order to ensure the mission's success, battery power must be managed efficiently. With newly working code for components in the "header stack", operating and power tests were conducted and results were recorded. These results were fairly consistent with power and current data given within component manuals. This data will be critical in the next stages of testing, where battery life will be limited and actions will need to be taken to ensure the CubeSat does not drain its battery in space.
\end{abstract}

\section{Introduction}

Beginning in 2016, the Carthage College CubeSat team was selected by NASA to design and build a 3U CubeSat named CaNOP (Canopy Near-infrared Observing Project), funded through the WSGC. The overall mission of CaNOP is to demonstrate that large hyperspectral imaging capabilities on satellites (such as the Landsat missions) is possible in the efficient CubeSat form. From these multispectral images of global forest canopies, spectral ratios such as NDVI will be computed in efforts to infer carbon content in both old-growth and new-growth forests.

One major area of research in order to ensure mission success is power management. Power tests were conducted on operating components, and these results were analyzed to determine how battery power will be budgeted and managed during the CubeSat's mission. Advances in project software coding from last summer made these tests possible.

\section{Testing and Analysis Procedures}

In order to test the components in the flat-stack configuration, a power supply and multimeter were used to supply and measure voltages and currents. Wires were connected to the Electrical Power System component (EPS) from the power supply to provide over-voltage protection and power data. From there, the components that required testing were connected and powered. Three trials were conducted and power and current data was recorded from the EPS, power supply, and/or multimeter to ensure accuracy.

To properly analyze this data, external software and code was used to simulate mission modes of operation and environments. Alongside comparing the raw data to the provided estimates in the components' operation documents, Systems Tool Kit (STK), a physics-based software package from Analytical Graphics, Inc, was used to model the CaNOP's mission in space. STK was able to produce received solar power data that models expected power levels our CubeSat would 
experience in LEO. All of this power data was then ran through supplementary VPython code to create example mission power data, which consisted of the power discharge and charge wattages for a full 30 days of operation as CaNOP cycled through its various modes.

\section{Power Test Results}

After completion of the first stage of testing, the data provided nominal voltage and current measurements for each component tested. Voltage requirements varied between each component but the voltage levels that were expected and confirmed were performance in the $3.3 \mathrm{~V}, 5 \mathrm{~V}$, battery voltage $(7.6 \mathrm{~V})$, and $12 \mathrm{~V}$ circuits. Current draws were also nominal, consistent with the documentation.

Upon testing example solar cycles the CaNOP would experience during its mission, two dates and times were examined, one being an example of a poor solar cycle and the other an excellent solar cycle. STK data provided a poor charge cycle for the time period of (12/16/2018 7:03:55 AM UTCG) to (12/16/2018 8:03:55 AM UTCG), which resulted in the solar panels receiving an effective $3.12 \mathrm{Whr}$ of charge. STK provided an excellent charge cycle for the time period of (2/1/2019 12:44:00 PM UTCG) to (2/1/2019 1:44:00 PM UTCG), which resulted in the solar panels receiving an effective $6.55 \mathrm{Whr}$ of charge.

Analysis of the raw data and results of the VPython code resulted in estimate mission mode power draws. The results concluded that while in a standard "Charge" mode, the CaNOP CubeSat used 5.45 Whr. During image capture, with capture durations taken into consideration, the CaNOP CubeSat used $0.08 \mathrm{Whr}$. During image downlink to ground for a single data packet, with downlink durations taken into consideration, the CaNOP CubeSat uses 13.96 Whr. During "Idle" mode, where the nanosatellite conducts system checks and corrects its orientation, the CaNOP CubeSat uses 7.6 Whr.

\section{Interpretation of Results}

From the nominal current and voltage measurements of each component, we can infer that the components will draw power as expected and that power consumption calculations will be fairly accurate for the mission. These values provide power measurements for each mode of functioning, which closely represent how much power will be needed for current mission operations. The EPS has built-in current limiters that restrict how much current can flow per voltage circuit. These results show that current levels stay comfortably below these limits.

Given the two extremes for solar charge cycles, calculations can be made to estimate how long the CaNOP will need to charge and show the variability of that duration. For reference, the battery used by the CaNOP has a maximum charge of $30 \mathrm{Whr}$ and has a recommended DoD of $20 \%$. To prevent the battery from passing $24 \mathrm{Whr}(80 \%)$, the CaNOP operating software is set to switch to "Charge" mode once that battery level is reached, and will remain in that state until a 
battery level of $27 \mathrm{Whr}(90 \%)$ is reached. With that said, an issue arises when comparing power gained through charge cycles and power expended while in "Charge" mode. If charging cycles provide power between $3.12 \mathrm{Whr}$ to $6.55 \mathrm{Whr}$, it is clear to see that "Charge" mode itself is unsustainable for a majority of the mission, drawing $5.45 \mathrm{Whr}$. This infers that the CaNOP will not be able to sustain itself in its mission as it stands. The solution to this issue will be that certain components shall be turned off during "Charge" mode in order to effectively charge the battery. These components will primarily be attitude control components, such as the magnetorquers and the Attitude Determination and Control System (ADCS).

\section{Acknowledgements}

I would like to thank the NASA Wisconsin Space Grant Consortium, and the NASA Undergraduate Student Instrumentation Program (USIP). Work supported by NASA USIP Award \#NNX16A170A. I would also like to thank our mentor Dr. Crosby for his time and dedication to the CaNOP CubeSat project, as well as Jeremiah Munsen for providing code allowing me to further my work. 